\title{
ON WEAK SOLUTIONS OF SEMILINEAR HYPERBOLIC-PARABOLIC EQUATIONS
}

\author{
JORGE FERREIRA \\ Departamento de Matemática \\ Universidade Estadual de Maringá \\ Agência Postal UEM \\ 87020-900, Maringá-PR, BRAZIL
}

(Recelved February 12, 1993 and in revised form December 15,1995)

\begin{abstract}
In this paper we prove the existence and uniqueness of weak solutions of the mixed problem for the nonlinear hyperbolic-parabolic equation

$$
\left(K_{1}(x, t) u^{\prime}\right)^{\prime}+K_{2}(x, t) u^{\prime}+A(t) u+F(u)=f
$$

with null Dirichlet boundary conditions and zero initial data, where $F(s)$ is a continuous function such that $s F(s) \geq 0, \forall s \in R$ and $\{A(t) ; t \geq 0\}$ is a family of operators of $L\left(H_{0}^{1}(\Omega) ; H^{-1}(\Omega)\right.$ ) For the existence we apply the Faedo-Galerkin method with an unusual a priori estimate and a result of W A Strauss Uniqueness is proved only for some particular classes of functions $F$
\end{abstract}

KEY WORDS AND PHRASES: Weak solutions, semilinear hyperbolic-parabolic equation, degenerating equations

1991 AMS SUBJECT CLASSIFICATION CODE: $35 \mathrm{~L} 60$.

\section{INTRODUCTION}

In this paper we study the global existence and uniqueness of weak solutions to the mixed problem for the nonlinear hyperbolic-parabolic equations

$$
(P)\left\{\begin{array}{l}
\left(K_{1}(x, t) u^{\prime}\right)^{\prime}+K_{2}(x, t) u^{\prime}+A(t) u+F(u)=f \text { in } Q \\
u(x, 0)=u_{0}(x), u^{\prime}(x, 0)=u_{1}(x), x \in \Omega
\end{array}\right.
$$

where $Q$ is a cylindrical domain of $R^{n+1}$ and $K_{1}(x, t), K_{2}(x, t), F$ are functions which satisfy some appropriate conditions

Physical motivations for studying $(P)$ come from several problems of continuum mechanics, such as turbulence, combustion, material aging, transonic flows, etc

Let $\Omega$ be a bounded open set in $R^{n}$ By $Q$ we represent the cylinder $\left.\Omega \times\right] 0, T[, T$ an arbitrary positive real number In $Q$ we consider the mixed problem for the hyperbolic-parabolic equation

$$
K_{1}(x) u^{\prime \prime}+K_{2}(x) u^{\prime}-\Delta u=f \text { in } Q
$$

where $K_{1}(x) \geq 0$ and $K_{2}(x) \geq \beta>0, x \in \Omega$

This type of equation was studied by Bensoussan-Lions-Papanicolau in [1] Medeiros [2] studied the existence of weak solution of the mixed problem for (1 1) plus the nonlinear term $|u|^{p} u, \rho>0$ Lima [3] analyzed the equation (1 11$)$ in a nonlinear abstract framework In Lar'kin [4] (1 1 1) was studied with more general nonlinearities, $K_{1}$ and $K_{2}$ depends also $t$, included also in $f$, but still with null initial conditions, plus strong restrictions on $f$

Many authors studied the equation (1 11$)$ when coefficients $K_{1}$ and $K_{2}$ also depend on $t$ Among them we mention Bryukhanov [5], Bubnov [6], Vragov [7] and Gadzhier [8] All of them assume zero initial data 
A significant nonlinear generalization of problem $\left(\begin{array}{ll}1 & 1\end{array}\right)$ is the following

$$
\left(K_{1}(x, t) u^{\prime}\right)+K_{2}(x, t) u^{\prime}-\Delta u+F(u)=f \text { in } Q
$$

with initial data

$$
u(x, 0)=u_{0}(x), u^{\prime}(x, 0)=u_{1}(x), x \in \Omega .
$$

Strauss [8] studied the existence of weak solution for ( 12 ) and (13) when $K_{1}=1, K_{2}=0$ and $F$ is a function that satisfies

$$
F \text { continuous and } s F(s) \geq 0 \text { for all } s \text { in } R \text {. }
$$

Maciel [10] studied existence and uniqueness of weak solutions problem (1 2)-(1 3), when $F$ is continuous and $s F(s) \geq 0$ for all $s \in R$, where the uniqueness is proved only for some particular cases of function $F$, and $K_{1}$ and $K_{2}$ satisfies

$$
\begin{aligned}
& K_{1}(x, t) \geq 0, \quad K_{2}(x, t) \geq \beta>0 . \\
& K_{2}(x, t)-\frac{1}{2}\left|K_{1 t}(x, t)\right| \geq \delta_{0}>0 .
\end{aligned}
$$

But with null initial conditions

The problem (1 2) may be included in the following general formulation

$$
\left(K_{1}(x, t) u^{\prime}\right)^{\prime}+K_{2}(x, t) u^{\prime}-\sum_{\imath, \jmath=1}^{n} \frac{\partial}{\partial x_{\imath}}\left(a_{\imath \jmath}(x, t) \frac{\partial u}{\partial x_{\jmath}}\right)+F(u)=f \text { in } Q .
$$

Observe that on the set $K_{1}(x, t)=0$ the equation (1 7) degenerate into parabolic equation

In this paper we study existence and uniqueness of weak solution of the mixed problem for the equation (1 7) in the case of null initial data, with $F$ satisfying condition (1 4) For the existence we apply the Faedo-Galerkin method (see Lions [11]), a priori estimates not usual and a result of W A Strauss for the nonlinear term (see Strauss [9]). The uniqueness is considered only for some particular cases of $F$ which permit the application of a method due to Visik and Ladyzenskaya [12]

The paper is organized as follows.

2 Some terminology and assumptions.

3 Existence of weak solutions

4 Uniqueness

\section{SOME TERMINOLOGY AND ASSUMPTIONS}

By $D(\Omega)$ we denote the space of infinitely differential functions with compact support contained in $\Omega$, the inner product and norm in $L^{2}(\Omega)$ and $H_{0}^{1}(\Omega)$ will be represented by $(.,),.|$.$| and ((.,)),.\|$. respectively By $H^{-1}(\Omega)$ we denote the dual space of $H_{0}^{1}(\Omega)$

Let $X$ be a Banach space, we denote by $L^{p}(0, T ; X), 1 \leq p<\infty$, the Banach space of vector-valued functions $u:(0, T) \rightarrow X$ which are measurable and $\|u(t)\|_{X}^{p} \in L^{p}(0, T)$ with the norm

$$
\|u\|_{L^{\infty}(0, T ; X)}=e s s \sup _{0<t<T}\|u(t)\|_{X} .
$$

Let us consider the following family of operators in $L\left(H_{0}^{1}(\Omega), H^{-1}(\Omega)\right)$

$$
A(t)=-\sum_{\imath, \jmath=1}^{n} \frac{\partial}{\partial x_{\imath}}\left(a_{\imath \jmath}(x, t) \frac{\partial}{\partial x_{\jmath}}\right)
$$

where

$$
\begin{gathered}
a_{\imath \jmath}=a_{\jmath \imath} \text { for all } i, \jmath=1, \ldots, n \\
\left\{a_{\imath \jmath}, \frac{\partial}{\partial t} a_{\imath \jmath}\right\} \in\left(L^{\infty}\left(0, T ; L^{\infty}(\Omega)\right)\right)^{2} \text { for all } i, j=1, \ldots, n .
\end{gathered}
$$

Here $\frac{\partial}{\partial t}$ denote the derivative in distributional sense 
We suppose that

$$
\sum_{i, j=1}^{n} a_{\imath \jmath}(x, t) \xi_{2} \xi_{\jmath} \geq \beta\left(\left|\xi_{1}\right|^{2}+\ldots+\left|\xi_{n}\right|^{2}\right),
$$

for all $(t, \xi) \in[0, T] \times R^{n}$ and a.e. in $\Omega$, with $\beta>0$ a constant. have

If we denote by $a(t, u, v)$ the family of bilinear forms in $H_{0}^{1}(\Omega) \times H_{0}^{1}(\Omega)$ associated with $A(t)$, we

$$
a(t, u, v)=\sum_{\imath, \jmath=1}^{n} \int_{\Omega} a_{\imath \jmath}(x, t) \frac{\partial u}{\partial x_{\imath}} \frac{\partial v}{\partial x_{\jmath}} d x,
$$

which is symmetric. From (2.3) it follows that

$$
a(t, u, u) \geq \beta\|u\|^{2}, \quad \text { for all } \quad u \in H_{0}^{1}(\Omega) \text { and } t \in[0, T] .
$$

From the assumptions on $a_{\imath j}$ we have that $h(t)=a(t, u, v)$ belongs to $L^{\infty}(0, T)$. It follows that

$$
h^{\prime}(t)=\sum_{\imath, \jmath=1}^{n} \int_{\Omega} a_{\imath \jmath}^{\prime}(x, t) \frac{\partial u}{\partial x_{\imath}} \frac{\partial v}{\partial x_{\jmath}} d x,
$$

which will be denoted by $a^{\prime}(t, u, v)$.

\section{EXISTENCE OF WEAK SOLUTIONS}

THEOREM 3.1. Consider $F$ satisfying (1.4) and suppose the functions $K_{1}, K_{2}$ and $f$ satisfy

$$
\begin{gathered}
K_{1}(x, t) \geq 0 \quad \text { a.e. in } \Omega, \quad \forall t \in[0, T] \\
\left\{K_{1}, K_{2}\right\} \in C^{1}\left([0, T]: L^{\infty}(\Omega)\right) \times C^{0}\left([0, T]: L^{\infty}(\Omega)\right), \\
K_{2}(x, t)+\frac{K_{1}^{\prime}(x, t)}{2} \geq \delta_{0}>0, \quad \text { a.e. in } \Omega, \quad \forall t \in[0, T], \\
f \in L^{2}\left(0, T ; L^{2}(\Omega)\right) .
\end{gathered}
$$

Then there exists $u$ defined in $Q$ such that

$$
\begin{gathered}
u \in L^{\infty}\left(0, T ; H_{0}^{1}(\Omega)\right), \\
\left\{u^{\prime}, \sqrt{\left(K_{1}\right)} u^{\prime}\right\} \in L^{2}(0, T ;(\Omega)) \times L^{\infty}\left(0, T ; L^{2}(\Omega)\right), \\
\left(K_{1} u^{\prime}\right)^{\prime}+K_{2} u^{\prime}+A(t) u+F(u)=f \quad \text { in } L^{1}\left(0, T ; H^{-1}(\Omega)+L^{1}(\Omega)\right),
\end{gathered}
$$

and

$$
u(0)=\left(K_{1} u^{\prime}\right)(0)=0
$$

REMARK 3.1. Suppose we have proved (3 5)-(3.7). Let us see that the initial data (3 8) make sense. In fact, by (3.5) and (3.6) follows, that $u \in C^{0}\left([0, T] ; L^{2}(\Omega)\right)$, therefore $u(0)$ makes sense By (3 6), (3.7) it follows that

$$
K_{1} u^{\prime} \in C^{0}\left([0, T] ; H^{-1}(\Omega)+L^{1}(\Omega)\right)
$$

so that $\left(K_{1} u^{\prime}\right)(0)=0$ also makes sense.

The proof of Theorem 31 will be a consequence of the following

THEOREM 3.2. Let $F_{k}: R \rightarrow R$ be a function satisfying the following conditions $s F_{k}(s) \geq 0$, $\forall s \in R, F_{k}$ is Lipschitzian and differentiable except on a finite number of points Then for each $k \in N$ there exists $u_{k}$ defined in $Q$ such that

$$
\left\{u_{k}, u_{k}^{\prime}, \sqrt{\left(K_{1}\right)} u_{k}^{\prime}\right\} \in L^{\infty}\left(0, T ; H_{0}^{1}(\Omega)\right) \times L^{2}\left(0, T ; L^{2}(\Omega)\right) \times L^{\infty}\left(0, T ; L^{2}(\Omega)\right),
$$


and

$$
\begin{gathered}
\left(K_{1} u_{k}^{\prime}\right)^{\prime}+K_{2} u_{k}^{\prime}+A(t) u_{k}+F_{k}\left(u_{k}\right)=f \text { in } L^{2}\left(0, T ; H^{-1}(\Omega)\right) \\
u_{k}(0)=\left(K_{1} u_{k}^{\prime}\right)(0)=0 .
\end{gathered}
$$

PROOF. We know (see [9]) that there exists a sequence of functions $F_{k}: R \rightarrow R$ such that each $F_{k}$ is Lipschitzian with constant $\alpha_{k}$, derivable except on a finite number of points. $s F_{k}(s) \geq 0$ and the sequence converges uniformly to $F$ on the bounded sets of $R$.

For each $k \in N, \epsilon \in R$ (fix) with $0<\epsilon<1$, we consider the problem

$$
\left(P_{k}\right)\left\{\begin{array}{l}
\left(K_{1 \epsilon} u_{\epsilon k}^{\prime}\right)^{\prime}+K_{2} u_{\epsilon k}^{\prime}+A(t) u_{\epsilon k}+F_{k}\left(u_{\epsilon k}\right)=f \\
u_{\epsilon k}(0)=\left(K_{1 \epsilon} u_{\epsilon k}^{\prime}\right)(0)=0
\end{array}\right.
$$

where $K_{1 \epsilon}=K_{1}+\epsilon$

Let $\left(w_{\imath}\right)_{\imath \in N}$ be a basis of $H_{0}^{1}(\Omega)$ and $V_{m}=\left[w_{1}, \ldots, w_{m}\right]$ the subspace generated by the $m$ first vectors of the basis $\left(w_{2}\right)$. For $m \in N$ consider the function

$$
u_{\epsilon k m}(x, t)=\sum_{\imath=1}^{n} g_{\epsilon k m i}(t) w_{\imath}(x), \quad 0 \leq t \leq t_{m}, \quad t_{m}<T,
$$

where $g_{\epsilon k m}(t)$ are found as solutions of the initial value problem for the system of ordinary differential equations

$$
\begin{gathered}
\left(\left(K_{1 \epsilon} u_{\epsilon k m}^{\prime}\right)^{\prime}, w_{\imath}\right)+\left(K_{2} u_{\epsilon k m}^{\prime}, w_{\imath}\right)+a\left(t, u_{\epsilon k m}, w_{\imath}\right) \\
+\left(F_{k}\left(u_{\epsilon k m}\right), w_{\imath}\right)=\left(f, w_{\imath}\right), 1 \leq i \leq m \\
u_{\epsilon k m}(0)=\left(K_{1} u_{\epsilon k m}^{\prime}\right)=0 .
\end{gathered}
$$

By Carathéodory's theorem follow the existence of $g_{\epsilon k m \imath}(t), 1 \leq i \leq m$. The a priori estimates which shall be obtained, permit us to extend the approximate solutions $u_{\epsilon k m}$ to the interval $[0, T]$ and also pass to the limit when $m, k \rightarrow \infty$ and $\epsilon \rightarrow 0$.

In fact, multiplying the equations (3.13) by $g_{\epsilon k m \imath}^{\prime}$, adding from $i=1$ to $i=m$ and integrating in $[0, T], t<t_{m}$, we have

$$
\begin{aligned}
& \left|\sqrt{K_{1 \epsilon}(t)} u_{\epsilon k m}^{\prime}(t)\right|^{2}+a\left(t, u_{\epsilon k m}(t), u_{\epsilon k m}(t)\right) \\
& +2 \int_{0}^{t}\left(\left(K_{2}(s)+\frac{1}{2} K_{1}^{\prime}(s)\right) u_{\epsilon k m}^{\prime}(s), u_{\epsilon k m}^{\prime}(s)\right) d s+2 \int_{0}^{t}\left(F_{k}\left(u_{\epsilon k m}(s), u_{\epsilon k m}^{\prime}(s)\right)\right) d s \\
& =2 \int_{0}^{t}\left(f, u_{\epsilon k m}^{\prime}(s)\right) d s+\int_{0}^{t} a^{\prime}\left(s, u_{\epsilon k m}(s), u_{\epsilon k m}(s)\right) d s .
\end{aligned}
$$

REMARK 3.2. Let $G_{k}(t)=\int_{0}^{t} F_{k}(\xi) d \xi$. Then $G_{k}(t) \geq 0$ and

$$
\int_{0}^{t}\left(F_{k}\left(u_{\epsilon k m}(s)\right), u_{\epsilon k m}^{\prime}(s)\right)=\int_{\Omega} G_{k}\left(u_{\epsilon k m}(x, t)\right) d x \geq 0 .
$$

Using Remark 3.2 and hypotheses (2.4), (3.2) and (3.3) in (3.15) we obtain the estimate

$$
\left|\sqrt{K_{1 \epsilon}(t)} u_{\epsilon k m}^{\prime}(t)\right|^{2}+\delta_{0} \int_{0}^{t}\left|u_{\epsilon k m}^{\prime}(s)\right|^{2}+\beta\left\|u_{\epsilon k m}(t)\right\|^{2} \leq C,
$$

where $C$ is a constant independent of $t, k, \epsilon$, and $m$. The estimate (3.16) implies that we can prolongate the approximate solution $u_{\epsilon k m}$ to interval $[0, T]$. Then there exists a subsequence of $\left(u_{\epsilon k m}\right)$, which we still denote by $\left(u_{\epsilon k m}\right)$, and a function $u_{\epsilon k}$ such that

$$
\begin{aligned}
u_{\epsilon k m} & \rightarrow u_{\epsilon k} \quad \text { weakly-star in } L^{\infty}\left(0, T ; H_{0}^{1}(\Omega)\right) \\
u_{\epsilon k m}^{\prime} & \rightarrow u_{\epsilon k}^{\prime} \quad \text { weakly in } L^{2}\left(0, T ; L^{2}(\Omega)\right) \\
\sqrt{K_{1 \epsilon}} u_{\epsilon k m}^{\prime} & \rightarrow \sqrt{K_{1 \epsilon}} u_{\epsilon k}^{\prime} \quad \text { weakly-star in } L^{\infty}\left(0, T ; L^{2}(\Omega)\right) .
\end{aligned}
$$


REMARK 3.3. We have that $F_{k}$ is Lipschitzian with constant $\alpha_{k}>0$, and satisfy $s F_{k}(s)>0 \forall s \in R$, then $F_{k}(0)=0$ Therefore we obtain

$$
\begin{aligned}
\left|F_{k}\left(u_{\epsilon k m}\right)\right|_{L^{2}\left(0, T, L^{2}(\Omega)\right)}= & \left(\int_{0}^{T}\left|F_{k}\left(u_{\epsilon k m}(t)\right)\right|^{2}\right)^{\frac{1}{2}} \leq\left(\alpha_{k}^{2} \int_{0}^{T}\left|u_{\epsilon k m}(t)\right|^{2} d t\right)^{\frac{1}{2}} \\
& \leq \alpha_{k}\left|u_{\epsilon k m}\right|_{L^{2}\left(0, T, L^{2}(\Omega)\right)} .
\end{aligned}
$$

Using (3 16) and the continuous inclusion of $L^{\infty}\left(0, T ; L^{2}(\Omega)\right)$ into $L^{2}\left(0, T ; L^{2}(\Omega)\right)$, we obtain that

$$
\left|F_{k}\left(u_{\epsilon k m}\right)\right|_{L^{2}\left(0, T, L^{2}(\Omega)\right)} \leq C\left(\alpha_{k}\right)
$$

where $C\left(\alpha_{k}\right)$ is a constant which depends only on $\alpha_{k}$. Then, by the compactness argument, it follows that

$$
F_{k}\left(u_{\epsilon k m}\right) \rightarrow F_{k}\left(u_{\epsilon k}\right) \text { a e in } Q, \text { as } m \rightarrow \infty .
$$

Now we combine Remark 33 with a result from Lions (see [11, p 12]), we have that

$$
F_{k}\left(u_{\epsilon k m}\right) \rightarrow F_{k}\left(u_{\epsilon k}\right) \text {, weakly in } L^{2}\left(0, T ; L^{2}(\Omega)\right) .
$$

Multiplying (3 13) by $\theta \in C_{0}^{\infty}(\Omega)$, integrating in $[0, T]$, using (3 17)-(3 19) and the fact $\left(w_{\imath}\right)$ is a basis of $H_{0}^{1}(\Omega)$ we have

$$
\begin{array}{r}
-\int_{0}^{T}\left(K_{1 \epsilon} u_{\epsilon k}^{\prime}, v \theta^{\prime}\right) d t+\int_{0}^{T}\left(K_{2} u_{\epsilon k}^{\prime}, v \theta\right) d t+\int_{0}^{T} a\left(t, u_{\epsilon k}, v \theta\right) d t \\
+\int_{0}^{T}\left(F_{k}\left(u_{\epsilon k}\right), v \theta\right) d t=\int_{0}^{T}(f, v \theta) d t, \forall v \in H_{0}^{1}(\Omega)
\end{array}
$$

Observe that the estimate ( 316 ) is yet true for $u_{\epsilon k}$ Then we can take the limit as $\epsilon \rightarrow 0$ in (3 21) and we obtain a function $u_{k}$, such that

$$
\left\{u_{k}, u_{k}^{\prime}, \sqrt{K_{1}} u_{k}^{\prime}\right\} \in L^{\infty}\left(0, T ; H_{0}^{1}(\Omega)\right) \times L^{2}\left(0, T ; L^{2}(\Omega)\right) \times L^{\infty}\left(0, T ; L^{2}(\Omega)\right)
$$

and

$$
\left(K_{1} u_{k}^{\prime}\right)^{\prime}+K_{2} u_{k}+A(t) u_{k}+F_{k}\left(u_{k}\right)=f \quad \text { in } \quad L^{2}\left(0, T ; H^{-1}(\Omega)\right) .
$$

By the standard way (as in Lions [11]) we can see that

$$
u_{k}(0)=\left(K_{1} u_{k}^{\prime}\right)(0)=0 .
$$

The proof of Theorem 3.2 is completed.

Multiplying (3.23) by $u_{k}$ (in the sense of the duality between $H^{-1}(\Omega)$ and $H_{0}^{1}(\Omega)$ ) and integrating in $[0, T]$ we obtain

$$
\int_{0}^{T}\left(F_{k}\left(u_{k}\right), u_{k}\right) d t \leq C
$$

where $C$ is a constant which does not depend on $k$

Observe that the estimate $(316)$ does not also depend on $k$. This implies that there exists a subsequence of $\left(u_{k}\right)_{k \in N}$, which we still denote by $\left(u_{k}\right)$, and a function $u$ such that

$$
\begin{aligned}
u_{k} & \rightarrow u \text { weakly-star in } L^{\infty}\left(0, T ; H_{0}^{1}(\Omega)\right) \\
u_{k}^{\prime} & \rightarrow u^{\prime} \quad \text { weakly in } L^{2}\left(0, T ; L^{2}(\Omega)\right) \\
\sqrt{K_{1}} u_{k}^{\prime} & \rightarrow \sqrt{K_{1}} u^{\prime} \quad \text { weakly-star in } L^{\infty}\left(0, T ; L^{2}(\Omega)\right) .
\end{aligned}
$$

By (3 26) and (3.27) and Aubin-Lions' lemma (see [11, p 58]) we have that

$$
u_{k} \rightarrow u \text { strongly in } L^{2}\left(0, T ; L^{2}(\Omega)\right) \text { and a.e. in } Q .
$$

At this point we state the following theorem from Strauss 
STRAUSS' THEOREM. Let $\Omega$ be a finite measure space with measure $d x, X$ and $Y$ the real Banach spaces, $\left(u_{\jmath}\right)$ a sequence of strong measurable functions of $\Omega$ in $X$ and $\left(h_{\jmath}\right)$ a sequence of functions of $\Omega \times X$ such that

(i) $\left(h_{\jmath}\right)$ is uniformly bounded in $\Omega \times K$ for all bounded $K \subset X$.

(ii) $h_{\jmath}\left(., u_{\jmath}().\right)$ is measurable and $\int_{\Omega}\left\|u_{\jmath}(x)\right\|_{X}\left\|h_{\jmath}\left(x, u_{\jmath}(x)\right)\right\|_{Y} \leq C<\infty, \forall j$

(iii) $\left\|h_{\jmath}\left(x, u_{\jmath}(x)\right)-v(x)\right\|_{Y} \rightarrow 0$ as $j \rightarrow \infty$ a e. in $\Omega$.

Then $v \in L^{1}(\Omega ; Y)$ and

$$
\int_{\Omega}\left\|h_{\jmath}\left(x, u_{\jmath}(x)-v(x)\right)\right\|_{Y} \rightarrow 0 \quad \text { as } \quad j \rightarrow \infty .
$$

Since we have (3 25) and (3.29), we can use Strauss' Theorem and we have that

$$
F_{k}\left(u_{k}\right) \rightarrow F(u) \text { strongly in } L^{1}(Q) .
$$

Finally we take the limit as $\epsilon \rightarrow 0$ and $k \rightarrow \infty$ (3.21) and we obtain (3.7).

\section{UNIQUENESS}

We do not prove uniqueness in the general case. For some particular $F$ we can use Ladyzenskaya's methods (see [12]) in order to obtain the uniqueness of solutions So we have uniqueness in the following cases

THEOREM 4.1. Suppose that $F: R \rightarrow R$ is a local Lipschitz function such that $s F(s) \geq 0$ for all $s \in R, K_{2}^{\prime} \in L^{\infty}\left(0, T ; L^{2}(\Omega)\right)$ and

$$
K_{2}(x, t)-\frac{1}{2}\left|K_{1}^{\prime}(x, t)\right| \geq \delta_{0}>0 \quad \text { a.e. in } \quad \Omega, \forall t \in[0, T]
$$

and $n=1$. Then there exists a unique function $u(x, t),(x, t)$ in $Q$, such that

$$
\left\{u, u^{\prime}\right\} \in L^{\infty}\left(0, T ; H_{0}^{1}(\Omega)\right) \times L^{2}\left(0, T ; L^{2}(\Omega)\right)
$$

which is a solution of the problem (3.5)-(3.8).

PROOF. Suppose we have two solutions $u$ and $v$ in the conditions of Theorem 31 . It follows that $w=u-v$ is a solution of:

$$
\begin{gathered}
\left(K_{1} w^{\prime}\right)^{\prime}+K_{2} w^{\prime}+A(t) w+F(u)-F(v)=0 \text { in } L^{1}\left(0, T ; H^{-1}(\Omega)+L^{1}(\Omega)\right) \\
w(0)=\left(K_{1} w^{\prime}\right)(0)=0 .
\end{gathered}
$$

We prove that $w=0$ in $[0, T]$. With $n=1$ then $H_{0}^{1}(\Omega) \subset L^{\infty}(\Omega)$. Since $\{u, v\} \in\left(L^{\infty}\left(0, T ; H_{0}^{1}(\Omega)\right)\right)^{2}$ and $F$ is a local Lipschitz function then

$$
\{F(u), F(v)\} \in\left(L^{\infty}\left(0, T ; H^{-1}(\Omega)\right)\right)^{2} .
$$

Therefore by (4.3) we have

$$
\left(K_{1} w^{\prime}\right)^{\prime}+K_{2} w^{\prime}+A(t) w+F(u)-F(v)=0 \quad \text { in } \quad L^{2}\left(0, T ; H^{-1}(\Omega)\right) .
$$

We consider for $0<s<T$ a function $\Psi(t)$ be defined by:

$$
\Psi(t)=\left\{\begin{array}{l}
-\int_{t}^{s} w(\xi) d \xi \quad \text { if } 0 \leq t \leq s \\
0 \text { if } s<t \leq T .
\end{array}\right.
$$

This integral exists and $\Psi(t) \in H_{0}^{1}(\Omega)$ If we represent

$$
w_{1}(t)=\int_{0}^{t} w(\xi) d \xi
$$

then

$$
\Psi(t)=w_{1}(t)-w_{1}(s)
$$


We have $\Psi(s)=0, \Psi^{\prime}(t)=w(t)$, and it makes sense to evaluate $\left(K_{1} w^{\prime}\right) \in H^{-1}(\Omega)$ in $\Psi(t) \in H_{0}^{1}(\Omega)$. We obtain

$$
\begin{aligned}
\int_{0}^{s}\left\langle\left(K_{1} w^{\prime}\right)^{\prime}, \Psi(t)\right\rangle_{H^{-1}(\Omega), H_{0}^{1}(\Omega)} d t+\int_{0}^{s}\left(K_{2} w^{\prime}, \psi(t)\right) d t \\
+\int_{0}^{s}\langle A(t) w, \Psi(t)\rangle_{H^{-1}(\Omega), H_{0}^{1}(\Omega)} d t \\
+\int_{0}^{s}(F(u)-F(v), \psi(t))>d t=0
\end{aligned}
$$

so we have

$$
\begin{aligned}
\int_{0}^{s}\left\langle\left(K_{1} w^{\prime}\right)^{\prime}, \Psi(t)\right\rangle_{H^{-1}(\Omega), H_{0}^{1}(\Omega)} d t & =-\frac{1}{2}\left(\left(K_{1} w(s), w(s)\right)\right)+\frac{1}{2} \int_{0}^{s}\left(K_{1}^{\prime} w, w\right) d t \\
\int_{0}^{s}\left(\left(K_{2} w^{\prime}\right)(t), \Psi(t)\right) d t & =-\int_{0}^{s}\left(K_{2}^{\prime} w, \Psi(t)\right) d t-\int_{0}^{s}\left(K_{2} w, w\right) d t \\
\int_{0}^{s}\langle A(t) w, \Psi(t)\rangle_{H^{-1}(\Omega), H_{0}^{1}(\Omega)} d t & =\int_{0}^{s} a(t, \Psi(t), \Psi(t)) d t \\
& =-\frac{1}{2} a(0, \Psi(0), \Psi(0))-\frac{1}{2} \int_{0}^{s} a^{\prime}(t, \Psi(t), \Psi(t)) d t
\end{aligned}
$$

Then from Equation (4 6) we obtain that

$$
\begin{aligned}
& \frac{1}{2}\left(\left(K_{1} w\right)(s), w(s)\right)+\delta_{0} \int_{0}^{s}|w(t)|^{2} d t+\frac{\beta}{2}\|\Psi(0)\|^{2} \\
& \quad \leq\left|\int_{0}^{s}(F(u)-F(v), \Psi(t))\right| d t+\left|\int_{0}^{s}\left(K_{2}^{\prime} w, \Psi(t) d t\right)\right| \\
& \quad+\frac{1}{2} \int_{0}^{s}\left|a^{\prime}(t, \Psi(t), \Psi(t))\right| d t .
\end{aligned}
$$

With $\{u, v\} \in L^{\infty}\left(0, T ; L^{\infty}(\Omega)\right)^{2}$ then exist a constant $C>0$ such that

$$
|u(x, t)| \leq C \text { and }|v(x, t) \leq C| \text { a e. in } Q .
$$

Therefore we have

$$
\begin{aligned}
\left|\int_{0}^{s}(F(u)-F(v), \Psi(t)) d t\right| \leq \int_{0}^{s} \int_{\Omega} \mid F(u(x, t))- & F(v(x, t))|| \Psi(t) \mid d x d t \\
& \leq \beta_{1} \int_{0}^{s}|w(t)||\Psi(t)| d t
\end{aligned}
$$

So we have

$$
\begin{gathered}
\left|\int_{0}^{s}\left(K_{2}^{\prime} w, \Psi(t)\right) d t\right| \leq C \int_{0}^{s}|w(t)||\Psi(t)| d t \\
\frac{1}{2} \int_{0}^{s}\left|a^{\prime}(t, \Psi(t), \Psi(t))\right| d t \leq C_{1} \int_{0}^{s}\left\|w_{1}(t)\right\|^{2} d t+C_{1} s\left\|w_{1}(s)\right\|^{2} .
\end{gathered}
$$

Using (4.9), (4.10) and (4.11) in (4.8) and the fact $\Psi(0)=-w_{1}(s)$ we obtain

$$
\begin{aligned}
& \frac{1}{2}\left(\left(K_{1} w\right)(s), w(s)\right)+\delta_{0} \int_{0}^{s}|w(t)|^{2} d t+\frac{\beta}{2}\left\|w_{1}(s)\right\|^{2} \\
& \leq C \int_{0}^{s}|w(t)|\left|w_{1}(s)\right| d t+C \int_{0}^{s}|w(t)|\left|w_{1}(s)\right| d t \\
&+C_{1} \int_{0}^{s}\left\|w_{1}(t)\right\|^{2} d t+C_{1} s\left\|w_{1}(s)\right\|^{2}
\end{aligned}
$$


Let $\lambda>0$ be a number to be fixed later. The inequality $(412)$ become:

$$
\begin{array}{r}
\frac{1}{2}\left(\left(K_{1} w\right)(s), w(s)\right)+\left(\delta_{0}-C^{2} \lambda\right) \int_{0}^{s}|w(t)|^{2} d t+ \\
\left(\frac{\beta}{2}-\left(C_{1}+\frac{C^{2}}{2 \lambda}\right)\right) s\left\|w_{1}(s)\right\|^{2} \\
\leq\left(C_{1}+\frac{C^{2}}{2 \lambda}\right) \int_{0}^{s}\left\|w_{1}(t)\right\|^{2} d t
\end{array}
$$

Now choosing $\lambda$ such that $\delta_{0}-C^{2} \lambda=\frac{\delta_{0}}{2}$ we obtain $\lambda=\frac{\delta_{0}}{2 C^{2}}$ and if $s_{0}$ is such that $\frac{\beta}{4}-\left(C_{1}+\frac{C^{2}}{2 \lambda}\right) s_{0}=\frac{\beta}{4}$ we obtain

and for $0 \leq s \leq s_{0}$,

$$
s_{0}=\frac{\beta}{4\left(C_{1}+\frac{C^{2}}{2 \lambda}\right)}>0
$$

$$
\left\|w_{1}(s)\right\| \leq \frac{4}{\beta}\left(C_{1}+\frac{C^{2}}{2 \lambda}\right) \int_{0}^{s}\left\|w_{1}(t)\right\|^{2} d t .
$$

This inequality implies $w_{1}(s)=0$ for all $0 \leq s \leq s_{0}$, or $w(s)=0$ on $0 \leq s \leq s_{0}$, or $w=0$ on $[0, T]$, which proves the uniqueness of Theorem 4.1.

THEOREM 4.2. If $n \geq 2, F \in C^{1}(R),\left|F^{\prime}(s)\right| \leq C|s|^{\rho}, \forall s \in R$, where $\frac{1}{n} \leq \rho \leq \frac{2}{n-2}$ if $n>2$ or $0<\rho<\infty$ if $n=2$. Then, there exists a unique function $u(x, t),(x, t) \in Q$ which is a solution of the problem (3.5)-(3.8).

PROOF. With $s F(s) \geq 0$ and $F$ is continuous then $F(0)=0$ and $\int_{0}^{s} F(\xi) d \xi \geq 0$ Then $F$ satisfies conditions of Mello [13], and therefore we have the uniqueness.

THEOREM 4.3. Suppose that $F: R \rightarrow R$ is a global Lipschitz function such that $s F(s) \geq 0$ for all $s \in R$ Then exists a unique function $u(x, t),(x, t) \in Q$ that is a solution of the problem (3.5)-(3.8).

PROOF. See Maciel [10]

ACKNOWLEDGMENT. The author is grateful to Professors L. A. Medeiros and Nirzi G Andrade for their valuable suggestions, to T F. Ma for reading the manuscript, and to the anonymous referee of the International Journal of Math. and Math. Sciences for his comments that improved this paper

\section{REFERENCES}

[1] BENSOUSSAN, A., LIONS, J. L. and PAPANICOLAU, G., Perturbations et augmentation des conditions initialies, Singular Perturbation and Boundary Lager Theory, Springer-Verlar Lyon (1976), 10-26.

[2] MEDEIROS, L. A., Non-linear hyperbolic-parabolic partial differential equation, Funkcialaj Ekvacioj, 23(02), (1980), 151-158.

[3] LIMA, O., Existence and Uniqueness of weak solutions of a hyperbolic-parabolic equation, Applicable Analysis, 24 (1987), 101-106.

[4] LAR'KIN, N A., Boundary problem in the large for a class of hyperbolic-parabolic equation, Sib. Math. J. 18 (06), (1977), 1003-1006.

[5] BRYUKHANOV, V.A., Mixed problem for a hyperbolic equation degenerated on a part of the boundary of a region, Differential Equations, 8 (1), (1972), 1-3.

[6] BUBNOV, B., Mixed problem for certain parabolic-hyperbolic equations, Differential Equatıons, 12 (3), (1976), 347-352.

[7] VRAGOV, N., On a mixed problem of a hyperbolic-parabolic equation, Dokl. Akad. Nauk. SSR, 224 (02), (1975), 1179-1183.

[8] GADZHIER M M, A mixed problem for a nonlinear hyperbolic-parabolic equation, Differentsial'nye Uraveniya, 13 (02), (1977), 185-289.

[9] STRAUSS, W A., On weak solutions of semilinear hyperbolic equation, An. Acad. Bras. Cienc. 42 (04), (1970), 645-651.

[10] MACIEL, A B., On a hyperbolic-parabolic equation with a continuous non-linearity, Nonlinear Analysis T. M. A., 20 (1993), 745-754.

[11] LIONS, J. L., Quelques méthodes des résolution des problèmes aux limites non-lineares, Dunod, Paris (1969).

[12] VISHIK, $M$ and LADYZENSKAYA, $O$., On boundary value problem for PDE and certain class of operators equations, Amer. Math. Soc. Transl. Ser. 2 (10), (1958)

[13] MELLO, E., Soluçoes fracas de um problema hiperbólico-parabólico não linear, Doctoral Thesis, IM-UFRJ, Rio de Janeiro, Brasil (1993). 


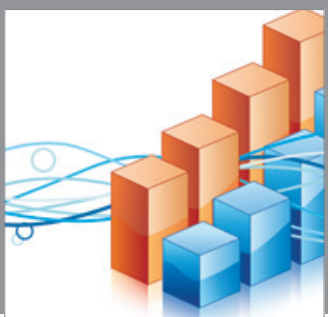

Advances in

Operations Research

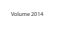

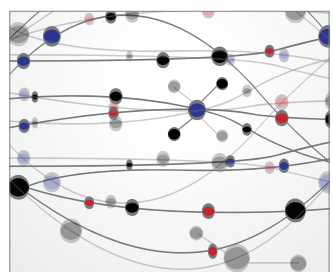

\section{The Scientific} World Journal
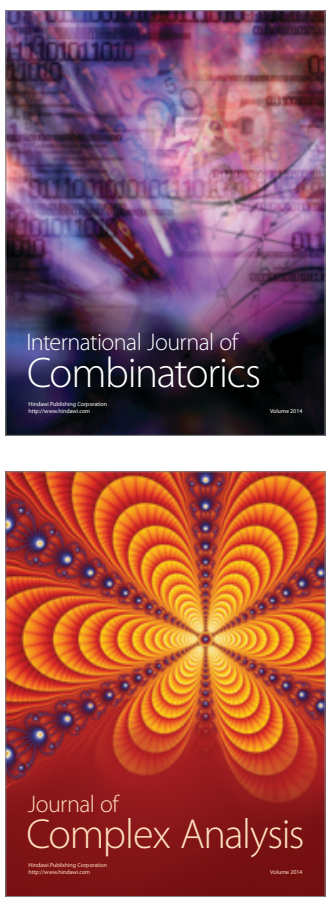

International Journal of

Mathematics and

Mathematical

Sciences
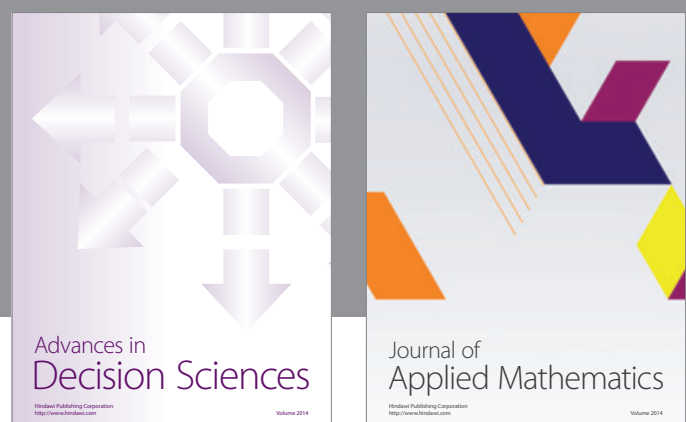

Journal of

Applied Mathematics
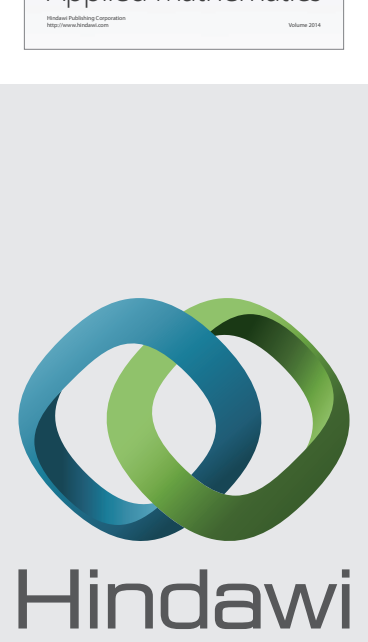

Submit your manuscripts at http://www.hindawi.com
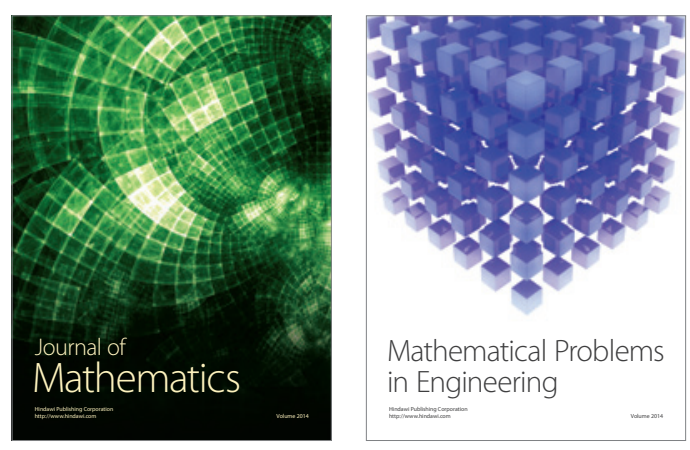

Mathematical Problems in Engineering
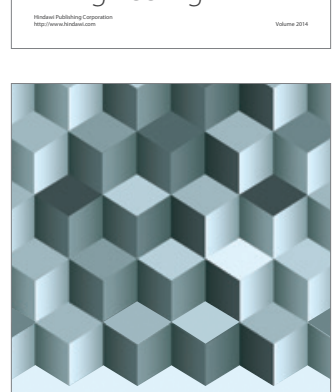

Journal of

Function Spaces
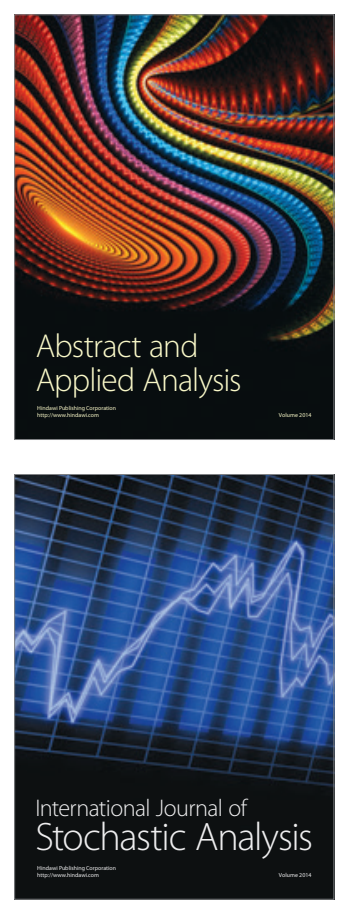

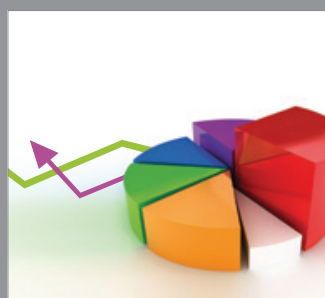

ournal of

Probability and Statistics

Promensencen
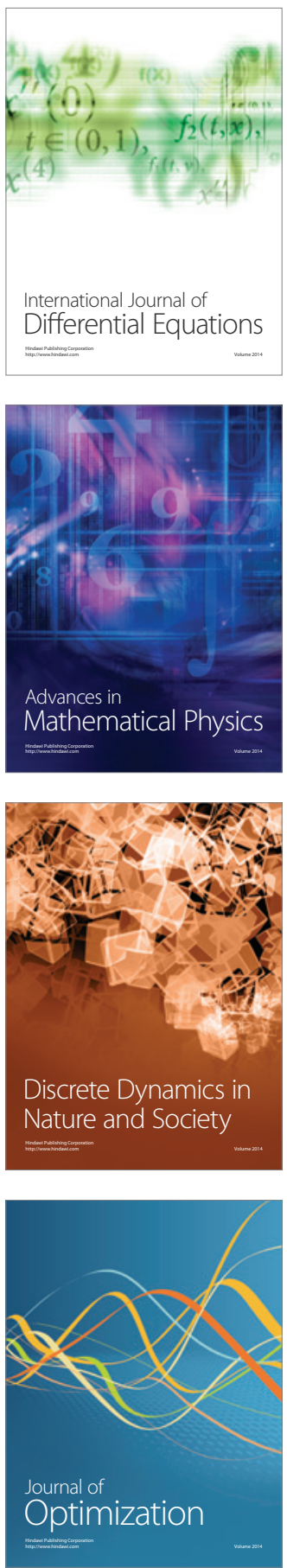